\title{
The Fundamentals of Unimodal Palmprint Authentication based on a Biometric System: A Review
}

\author{
Inass Shahadha Hussein ${ }^{*}$, Shamsul Bin Sahibuddin ${ }^{2}$, Nilam Nur Amir Sjarif ${ }^{3}$ \\ Faculty Razak of Technology and Informatics \\ UTM \\ Kuala Lumpur, Malaysia
}

\begin{abstract}
Biometric system can be defined as the automated method of identifying or authenticating the identity of a living person based on physiological or behavioral traits. Palmprint biometric-based authentication has gained considerable attention in recent years. Globally, enterprises have been exploring biometric authorization for some time, for the purpose of security, payment processing, law enforcement CCTV systems, and even access to offices, buildings, and gyms via the entry doors. Palmprint biometric system can be divided into unimodal and multimodal. This paper will investigate the biometric system and provide a detailed overview of the palmprint technology with existing recognition approaches. Finally, we introduce a review of previous works based on a unimodal palmprint system using different databases.
\end{abstract}

Keywords-Biometric system; palmprint; palmprint features; unimodal

\section{INTRODUCTION}

A biometric system is a technological system that uses information about a person (or another biological organism) to identify that person. Biometric systems rely on specific data about unique biological traits in order to work effectively [1]. Biometric systems can be applied to various applications such as criminal identification, car theft, laptop, and desktop authentication security systems, building access, locker protection, national security, military field, immigration, citizen identification, network access, attendance monitoring, and transaction processing system [2]. There are different types of biometric systems that have been employed by researchers. Examples of these types include biometric systems based on voice [3], DNA [4], keystroke [5], face [6], iris [7], hand geometry [8], fingerprint [9], gait [10] signature [11]. The development of palmprint recognition has helped humans perform tasks that were previously difficult or had to be carried out manually. Indirectly, these developments have helped humans by providing faster, more efficient and productive performance of tasks. Many researchers utilized the palmprint authentication system due to its reliable and confidential features [12], [13], [14].

The currently available literature shows that there have been a number of valuable reviews on the palmprint recognition system. However, finding a comprehensive study that is inclusive of various aspects of the palmprint recognition system is scarce. Therefore, this paper aims to investigate the biometric system and provide a review of the unimodal palmprint recognition system.

The paper is organized in the following sections. In Section 2 , the biometric system will be introduced. Section 3 comprises an overview of the palmprint technology. Section 4 will review previous works based on the unimodal palmprint system with using different databases. Finally, the paper is concluded in Section 5.

\section{BIOMETRIC SYSTEM}

Biometrics can be divided into physiological features and behavioral features. The physiological features include fingerprints, hand geometry, and palmprint. Fig. 1 shows the examples of physiological biometrics. The behavioral features comprise signatures, handwriting, and movements as illustrated in Fig. 2. These are unique features that exist in each individual and remain unchanged during a person's lifetime, hence providing a hopeful solution to the community.

Every trait has its metrics and limitations. For instance, the voice is less accurate, a keystroke needs a long observation time, and the face is affected by different poses, illumination, and aging factors. In addition, the iris sensor is very expensive, the hand geometry changes as children grow, the wearing of rings as well as rapid development of pregnant women in a short time, and the DNA is not user-friendly. Other limitations include the faint fingerprints of elderly persons and those involved in manual labors and even missing gait can be influenced by medical conditions, clothing, surface, footwear, and signature which are easy to forge $[14,15]$.

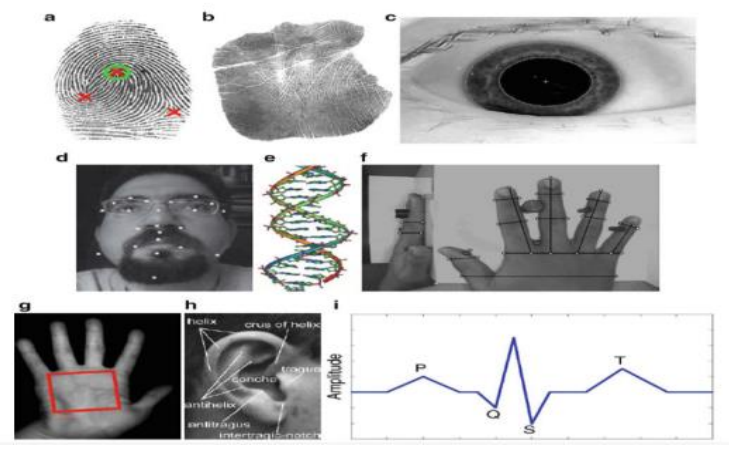

Fig. 1. Examples of Physiological Characteristics: a. Fingerprint, b. Palmprint, c. Iris, d. Face, e. DNA, g. Vein of hand, h. Ear and i. ECG. 


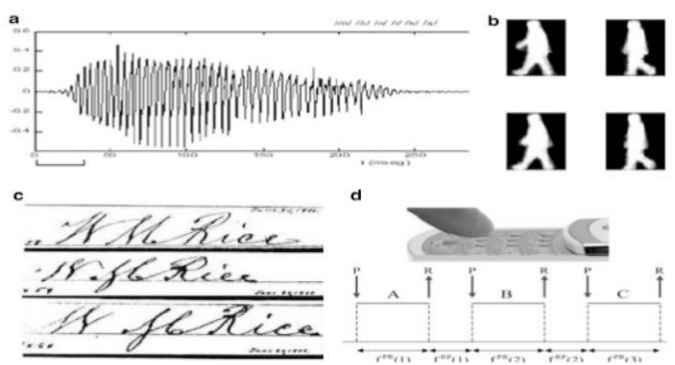

Fig. 2. Examples of Behavioral Characteristics: a. Voice, b. Gait, c. Signature and d. Keystroke.

\section{A. The Components of the Biometric System}

The main recognition components which are currently being used by the biometric system can be divided into five units [15-17]:

- Acquisitions unit: This unit depends on the biometric trait used. The sensor will be used to capture the trait which belongs to the user. The sensor captures an image, a voice signal or a frame sequence. The trait captured is called "sample."

- Segmentation unit: The sample region that contains the biometric information is isolated to produce the region of interest which will be used in the next unit.

- Features extraction unit: The features of the segmented sample are extracted, and a feature vector of the biometric trait is calculated. This vector is so suitable for the storage and analysis of databases through an information processing system. The vectors can be strings of bits or images or coordinates of specific points in the image signals or algebraic functions.

- Matching unit: The vector is compared to one or more of the prior-vectors which are stored in the database. The result of the matching unit is the "degree of matching," and the "similarity between the two comparators."

- Decision unit: The degree of matching is used to produce the final decision output of the biometric system. A threshold for the degree of matching value is used to convert the degree of matching to a logical decision, whether the comparison vectors belong to the same individual or not.

\section{B. Characteristics of the Biometric Traits}

The main characteristics of the biometric trait that must be taken into account $[15,18]$ are as follows:

- Universality: Everyone should possess the biometric trait.

- Distinctiveness: The individuals should be distinguished by the biometric trait.

- Permanence: Over time, the biometric trait should not change.

- Collectability: How easy it is to obtain biometrics.
- Performance: The speed and accuracy that can be acquired by using the biometric trait.

- Acceptance: The willingness of people to supply their biometric attributes for the process of recognition.

- Circumvention: The difficulty of piracy in the biometric system to avoid unauthorized access.

Table 1 illustrates a comparison of the characteristics of different biometric technologies [18].

TABLE I. DIFFERENT BIOMETRIC TECHNOLOGIES CHARACTERISTICS

\begin{tabular}{|c|c|c|c|c|c|c|c|}
\hline \multirow[b]{2}{*}{ Biometrics } & \multicolumn{7}{|c|}{ Characteristics } \\
\hline & 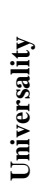 & 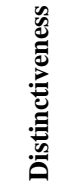 & 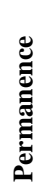 & 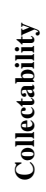 & 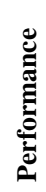 & 离 & \\
\hline $\begin{array}{l}\text { Facial } \\
\text { thermogram }\end{array}$ & $\mathrm{H}$ & $\mathrm{H}$ & $\mathrm{L}$ & $\mathrm{H}$ & M & $\mathrm{H}$ & $\mathrm{L}$ \\
\hline Hand vein & $\mathrm{M}$ & $\mathrm{M}$ & $\mathrm{M}$ & $\mathrm{M}$ & $\mathrm{M}$ & $\mathrm{M}$ & $\mathrm{L}$ \\
\hline Gait & $\bar{M}$ & $\mathrm{~L}$ & $\mathrm{~L}$ & $\mathrm{H}$ & $\mathrm{L}$ & $\mathrm{H}$ & $\mathrm{M}$ \\
\hline Keystroke & $\mathrm{L}$ & $\mathrm{L}$ & $\mathrm{L}$ & $\mathrm{M}$ & $\mathrm{L}$ & M & $\mathrm{M}$ \\
\hline Order & $\mathrm{H}$ & $\mathrm{H}$ & $\mathrm{H}$ & $\mathrm{L}$ & $\mathrm{L}$ & $\mathrm{M}$ & $\mathrm{L}$ \\
\hline Ear & M & $\mathrm{M}$ & $\mathrm{H}$ & $\mathrm{M}$ & $\mathrm{M}$ & $\mathrm{H}$ & $\mathrm{M}$ \\
\hline Hand geometry & $\mathrm{M}$ & $\mathrm{M}$ & $\mathrm{M}$ & $\mathrm{H}$ & $\mathrm{M}$ & $\mathrm{M}$ & $\mathrm{M}$ \\
\hline Finger & $\bar{M}$ & $\mathrm{H}$ & $\mathrm{H}$ & $\mathrm{M}$ & $\mathrm{H}$ & $\mathrm{M}$ & $\mathrm{M}$ \\
\hline Face & $\mathrm{H}$ & $\mathrm{L}$ & $\mathrm{M}$ & $\mathrm{H}$ & $\mathrm{L}$ & $\mathrm{H}$ & $\mathrm{H}$ \\
\hline Retina & $\mathrm{H}$ & $\mathrm{H}$ & $\mathrm{M}$ & $\mathrm{L}$ & $\mathrm{H}$ & $\mathrm{L}$ & $\mathrm{L}$ \\
\hline Iris & $\mathrm{H}$ & $\mathrm{H}$ & $\mathrm{H}$ & $\mathrm{M}$ & $\mathrm{H}$ & $\mathrm{L}$ & $\mathrm{L}$ \\
\hline Palmprint & $\mathrm{M}$ & $\mathrm{H}$ & $\mathrm{H}$ & $\mathrm{M}$ & $\mathrm{H}$ & $\mathrm{M}$ & $\mathrm{M}$ \\
\hline Voice & $\bar{M}$ & $\mathrm{~L}$ & $\mathrm{~L}$ & $\mathrm{M}$ & $\mathrm{L}$ & $\mathrm{H}$ & $\mathrm{H}$ \\
\hline Signature & $\mathrm{L}$ & $\mathrm{L}$ & $\mathrm{L}$ & $\mathrm{H}$ & $\mathrm{L}$ & $\mathrm{H}$ & $\mathrm{H}$ \\
\hline DNA & $\mathrm{H}$ & $\mathrm{H}$ & $\mathrm{H}$ & $\mathrm{L}$ & $\mathrm{H}$ & $\mathrm{L}$ & $\mathrm{L}$ \\
\hline
\end{tabular}

(H: high, M: medium, L: low)

Based on this comparison, we can see that the distinctiveness, permanence and performance characteristics for the palmprints are high. These are the main characteristics that make the palmprint a promising technology for authentication purposes.

\section{PALmprint TEChNOLOGY OVERVIEW}

The usefulness of palmprints has been progressively verified in the last fifteen years. A palmprint is defined as a small area on the surface of the palm which contains more 
information that can be very useful for a person's authentication system.

The images of palmprint contain unique characteristics of a reliable human identification making them a highly competitive feature in the palmprint recognition [19]. The studies of palmprint should utilize either the high resolution or low-resolution images. High-resolution images will be $500 \mathrm{dpi}$ or less, and these images are appropriate for forensic applications such as criminal detection. In most of the previous researches on palmprint, the main focus of recognition is on low-resolution images below 150 (dpi s), and these images are more appropriately used for civil and commercial applications such as access control [20, 21] [22] [23].

Furthermore, the palmprint image has a unique feature as it will be unchanged for a long period of time [24, 25]. Palmprint offers many distinctive features that can be used for precise recognition. The features of palmprint will be discussed in detail in the subsection below.

\section{A. Palmprint Image Features}

In general, the features of an image can be divided into two categories, global features, and local features. Global features remain a topic of interest in the past decade. Global means extracting the initial features of the whole image. Each pixel is taken into consideration [26]. The advantages of global features are they compress the size of representation and increase the speed of computation. However, the disadvantages are the variance of geometric distortion. Therefore, using global features alone is not efficient. Local features are considered the efficient representation of the image especially in the field of pattern recognition and computer vision. The local features are invariant to scale, translation and rotation [27]. Global features describe the visual content of the entire image using the vector. They represent texture, color, and shape information. Meanwhile, the local features aim to detect the interest points (IPs) in an image and describe them by a set of vectors [28]. Some examples of global palmprint descriptors are invariant moments ( $\mathrm{Hu}$ and Zernike) [29, 30] and histogram oriented gradients (HOC) [31]. For local palmprint descriptors, some of the examples are SIFT [32], SURF [33], LBP [34], BRISK [35] and MSER [36] . Fig. 3 represents the palmprint ink region while Fig. 4 represents the palmprint without ink region [37]. Meanwhile, the features of palmprint are shown in Table 2.

\section{B. Importance of Palmprint Technology}

Many researchers have highlighted the importance of palmprint system in their studies showing that palmprint has more distinctive features and availability of rich information such as lines, wrinkles, hills, dots, points, and texture. These unique characteristics offer different possibilities to represent the features of the palmprint and pattern recognition [19]. The sensors that are used to capture palmprint are low-cost with very low images of 75 dots per inch (dpi) [24]. The nature of palmprint which is anti-spoofing has made palmprint a reliable biometric feature. [24]. Among the various biometric features include the face, DNA, fingerprint, hand, iris, hand vein retina, sound and signature, palmprint has good potential as a personal identification method because it is fast and generally acceptable. In comparison to face recognition, palmprint is not affected by age and accessories. If compared to the recognition of a fingerprint, palm contains rich information and it only needs low-resolution image device capture that reduces the cost of the system. If compared to the recognition of the iris, images of the palmprint can be captured without intrusions as people may fear the side effects on their eyes.

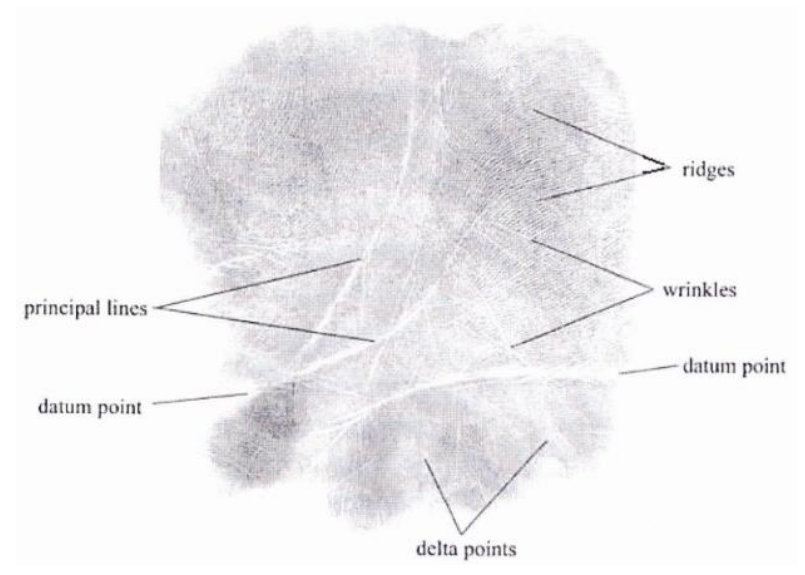

Fig. 3. Palmprint Features with ink.

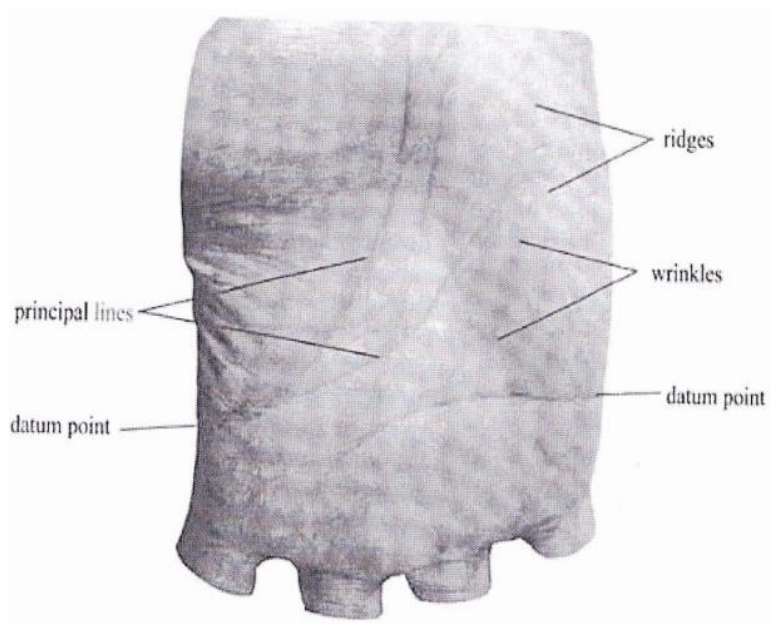

Fig. 4. Palmprint Features without ink.

TABLE II. MAIN FEATURES EXTRACTED FROM PALMPRINT

\begin{tabular}{|c|c|}
\hline Name of Feature & Explanation \\
\hline Geometry & $\begin{array}{l}\text { Depending on the shape of the hand; such as } \\
\text { height, width, and surface area }\end{array}$ \\
\hline Principle lines & Includes the position and location \\
\hline Datum points & $\begin{array}{l}\text { Extracting the two endpoints of the principal lines } \\
\text { provides a balance when recording the image. } \\
\text { Moreover, by defining Euclidean distance, } \\
\text { palmprint size will be obtained. }\end{array}$ \\
\hline Wrinkles & Small and shallower \\
\hline Minutiae & $\begin{array}{l}\text { Small and shallow points with various } \\
\text { measurements. }\end{array}$ \\
\hline Delta points & $\begin{array}{l}\text { Small regions which the center of the entire area. } \\
\text { They are similar to the shape of a delta. }\end{array}$ \\
\hline
\end{tabular}




\section{Palmprint System Authentication}

Authentication can be defined as giving the authorized persons the proper authority and access in proper time. Authentication is divided into identification and verification. These two different ways the biometric systems can be used according to the needs of the application. The identification phase can be performed once to several times by comparing the biometric data with each modal of the system database to detect the non-personal identity of the specified partition. The identification phase is very complicated and costs time, but it is very useful for negative recognition of critical issues such as in the context of forensic and criminal issues. However, in the phase of verification, the system confirms the identity of the individual already known by comparing the biometric data with the modal stored over in the database with that identity [38].

Verification needs less time since it is necessary to reduce the number of comparisons. Applications such as the security system for laptops or telephones, the presence system, security of access in electronic banking and offices are examples of the verification procedure [14]. Fig. 5 illustrates a typical palmprint authentication system.

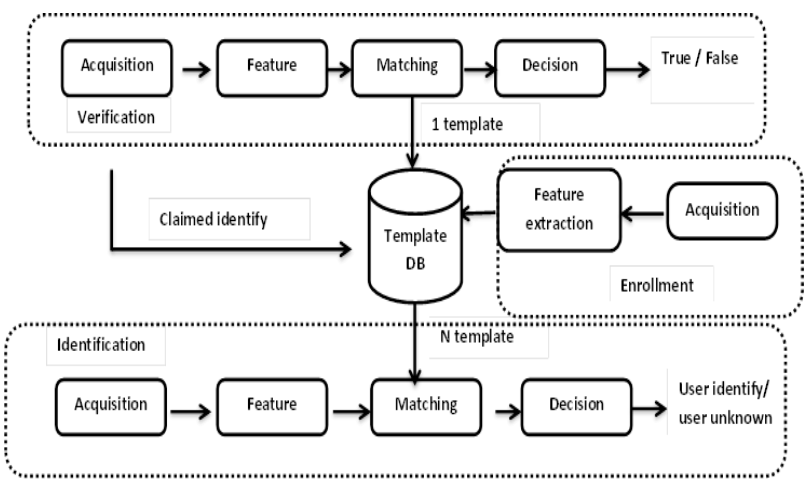

Fig. 5. A Typical Palmprint Authentication System.

As a result, palmprint authentication has the potential to achieve high accuracy for both identification and verification of humans [39]. The authentication of the palmprint of the hand is of great benefit to both civil and forensic applications since approximately $30 \%$ of the palmprint latent are raised from scenes of crime (i.e., wheels, steering, blades, etc.). For example, law implementation officials successfully use palmprint recognition to track down killers [38].

\section{REVIEW OF RECOGNITION APPROACHES OF PALMPRINT}

The approaches of palmprint matching and feature extraction are divided into three categories called holistic, local and hybrid. All of these approaches are explained in detail below.

\section{A. Holistic based Approaches}

Here, the original image will be used as the input of a holistic extractor or matcher. Table 3 summarizes the holistic approaches.

\section{B. Local based Approaches}

Local features refer to a pattern or distinct structure found in an image such as a point, edge, or small image patch. They are usually associated with an image patch that differs from its immediate surroundings by texture, color, or intensity. Some examples of local features are blobs, corners, and edge pixels. There are two groups of local features for palmprint recognition; ridges and creases points which can be extracted from high-resolution and low-resolution images.

Table 4 shows a summary of these approaches for local features. SIFT algorithm is one of the accurate methods used to extract local features. SIFT extractor is used for both lowresolution palmprint images and high-resolution images [23].

\section{Hybrid-Approach}

Hybrid approaches combine two or more of the recognition approaches to get high accuracy. For example, it could be a hybrid of two or three palmprint representations; Gabor line and subspace features LBP + 2DLPP [13] or a combination of the local features with holistic features [40].

\section{REVIEW OF PREVIOUS WORKS BASED UNIMODAL PALMPRINT SYSTEM}

The first system is called automated finger identification system (AFIS), built to support palmprint recognition in late 1990 by a Hungarian company. This is the first palmprint benchmark. Presently, a large number of researchers are investigating the palmprint technology by using different systems and various algorithms to improve this technology. The first system is unimodal which is then developed according to a multimodal with different criteria. The unimodal biometric system can be defined as using single trait for recognition. In the case of unimodal palmprint, it uses either the left or right palmprint. We will investigate the unimodal based on the following sections: a unimodal with various studies based contact based, contactless and high-resolution palmprint images.

TABLE III. HOLISTIC BASED APPROACHES

\begin{tabular}{|c|c|}
\hline The approaches & \multirow{2}{*}{ Work representation } \\
\hline Subspace method & \\
\hline $\begin{array}{l}\text { Unsupervised linear } \\
\text { method }\end{array}$ & PCA applications [13], ICA [41]. \\
\hline $\begin{array}{l}\text { Supervised linear } \\
\text { method }\end{array}$ & PCA + LDA [42] \\
\hline Kernel method & $\begin{array}{l}\text { Kernel PCA and kernel Fisher discriminant } \\
\text { applications }[43,44]\end{array}$ \\
\hline Tensor method & $\begin{array}{l}\text { 2DPCA [13] } \\
\text { 2D LPP [25] }\end{array}$ \\
\hline $\begin{array}{l}\text { Transform the } \\
\text { domain }\end{array}$ & Subspace methods in the transform domains[42] \\
\hline Invariant moments: & $\begin{array}{l}\text { Zernike moments [45] and Hu Invariant moments } \\
\text { [46] }\end{array}$ \\
\hline $\begin{array}{l}\text { Spectral } \\
\text { representation: }\end{array}$ & \multirow[t]{2}{*}{$\begin{array}{l}\text { Global statistical signatures in the wavelet domain } \\
\text { [47] }\end{array}$} \\
\hline Wavelet signature & \\
\hline Correlation filter & Advanced correlation filter [48] \\
\hline
\end{tabular}


TABLE IV. LOCAL BASED APPROACHES

\begin{tabular}{|c|c|}
\hline The approaches & \multirow[t]{2}{*}{ Work representation } \\
\hline Line based & \\
\hline Gaussian derivatives & $\begin{array}{l}\text { First and second class derivatives } \\
\text { Gaussian derivatives [49] }\end{array}$ \\
\hline Wide line detector & $\begin{array}{l}\text { Extract the location and width from } \\
\text { palmprint line [50] }\end{array}$ \\
\hline Hausdorff Distance & $\begin{array}{l}\text { Line segments Hausdorff Distance } \\
\text { application [51] }\end{array}$ \\
\hline Codec- Based & \multirow{2}{*}{$\begin{array}{l}2 \mathrm{D}-\text { Gabor code phase filter } \\
\text { responses [23] }\end{array}$} \\
\hline Code-Palm & \\
\hline Code- Fusion & $\begin{array}{l}\text { 2D-Gabor code phase filter } \\
\text { responses with the maximum } \\
\text { magnitude [52] }\end{array}$ \\
\hline Code- Orientation & $\begin{array}{l}\text { Orientation information coding of } \\
\text { palm lines [53] RLOC [54] }\end{array}$ \\
\hline Texture - descriptor & $\begin{array}{l}\text { Applications of LBP [55], DCT } \\
\text { Coefficients coding [56]. }\end{array}$ \\
\hline Scale-invariant feature & Application of SIFT [57] \\
\hline
\end{tabular}

\section{A. Review based Contact based Palmprint Database}

The popular poly $U$ database and Jiaotong Beijing University optical scanner are examples of contact based palmprint images. Fig. 6 illustrates the Poly U database image. There are two main approaches to extract features from the contact based images namely the line feature-based and orientation code based [23]. To extract the line feature either line or edge detectors such as DOG [58], Gabor filter [59], and radon filter [60] can be used. The DOG is sensitive to noise, illumination and it is difficult to distinguish the principal lines from wrinkles. On the other hand, to detect a line, we can detect radon transform (RT) which detects the intensity along potential lines that exist in the small local area. By developing RT and using a modified finite radon transform (MFRAT) [54], the summation of the image pixels over the lines is calculated. In general, the line based image does not achieve a very good result. Fig. 7 shows an example of the DOG line features and MFRAT line features.

Meanwhile, the orientation code based includes palm code [61], competitive code [62], fusion code [63], robust line orientation code (RLOC) [54], double orientation code (DOC) [64] and binary orientation co-occurrence vector (BOCV) [63]. Based on these approaches, a local orientation descriptor is used to represent palmprint images. In general, the orientation based method uses one or multiple orientations. Fig. 8 shows the examples of competitive code.

Furthermore, many algorithms for recognition are being developed over the years. Shang et al. [41] employed the artificial neural network in the palmprint classifier using the radial basis probabilistic neural network (RBPNN) and achieved a good result. Meanwhile, Raut and Humbe [65] suggested the extraction lines from palmprint biometric system by processing the morphological processes. This study discusses the importance of the exact location where the palmprint and shape of the hand images were extracted from a one-hand image acquired by a sensor.

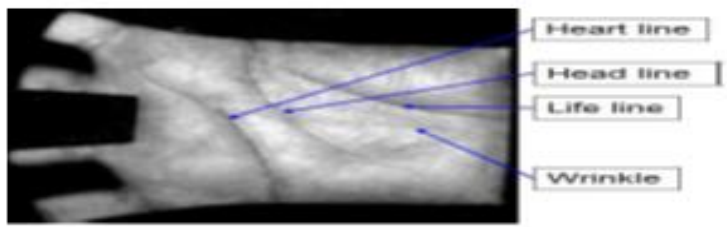

a.

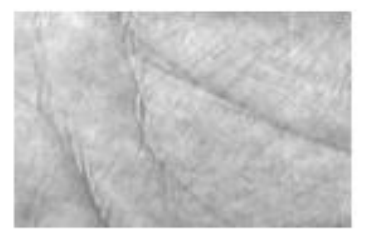

b.

Fig. 6. Poly U Database: a. Contact based Image, b. ROI.

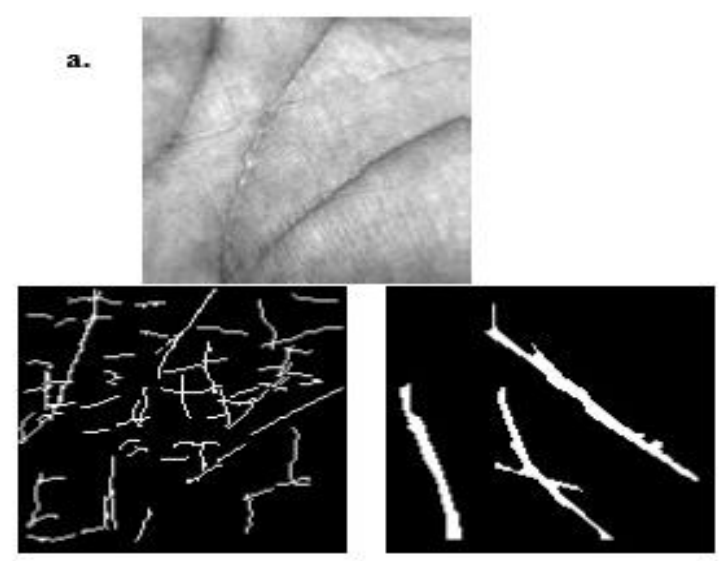

b.

c.

Fig. 7. Line Feature Detector a. ROI, b. DOG Line Features c. MFRAT Line Features.
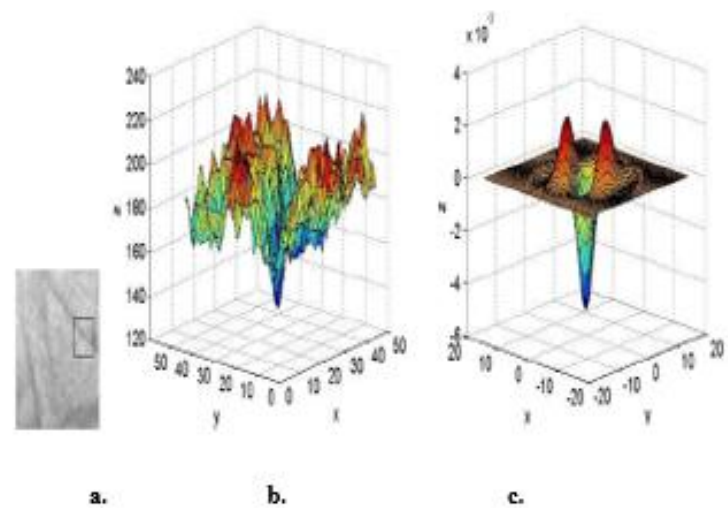

c.

Fig. 8. Examples of Competition: a. Sub-Image, b. The Intensity of Image, c. Gabor Filter with a Small Orientation. 
TABLE V. PREVIOUS WORKS BASED CONTACT BASED DATABASE

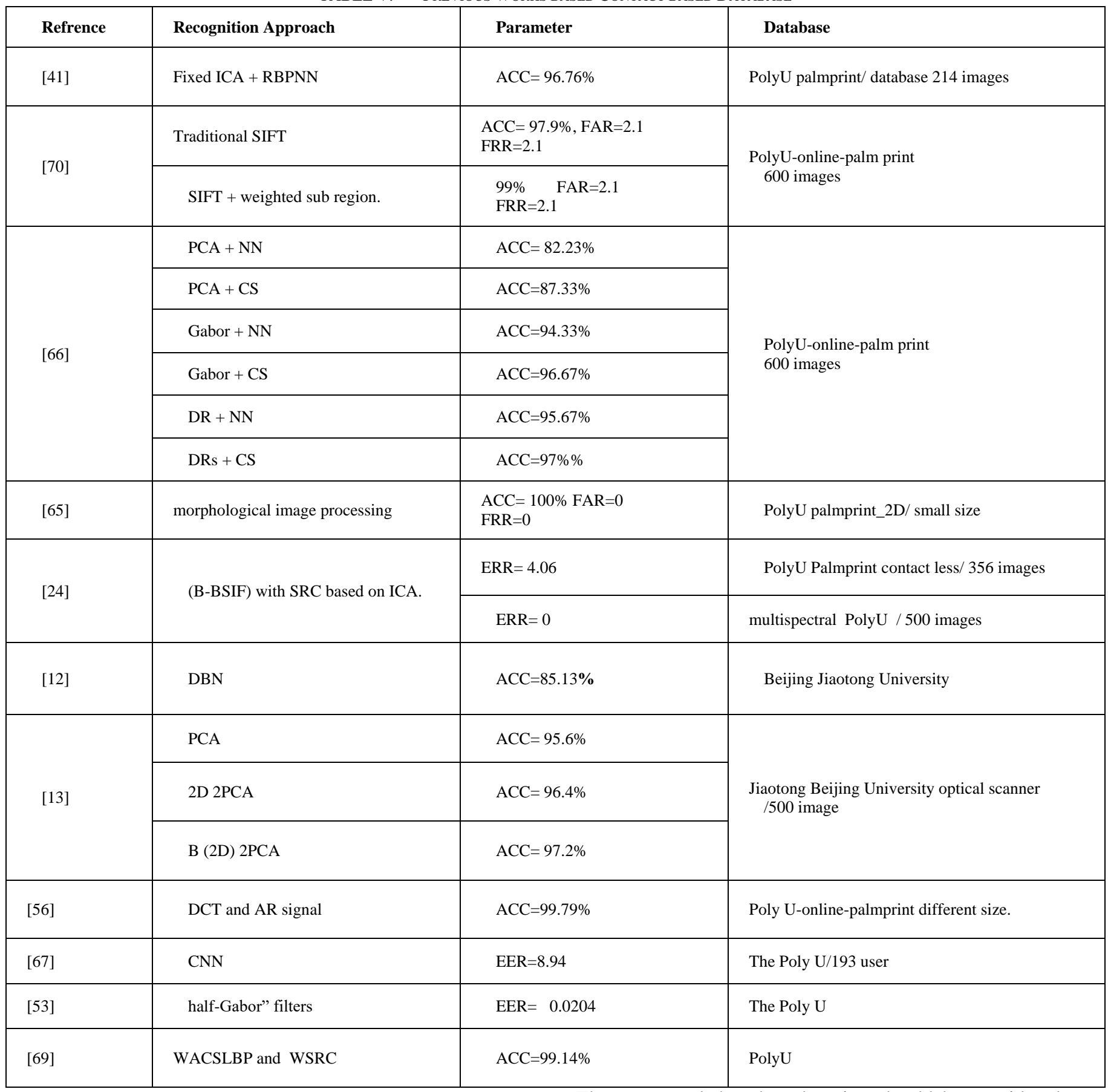

In a study conducted by $\mathrm{Li}$ et al.[66], they found that the solution to the problem of the illumination and noise of the image of palmprint recognition can be solved by proposing an approach to palmprint recognition through directional representations in low-quality conditions. In addition, they claimed that the Gabor algorithms are not robust for image recognition under uneven illumination and noise. Xin et al. [12] introduced deep learning into palmprint recognition involving three steps. First, a deep belief net (DBN) is built by top-to-down - unsupervised training. Second, the optimum parameters are chosen to build a robust performance. Finally, the testing sample is labeled using the DBN learning models.
They proposed that deep learning should be considered as a robust method of palmprint recognition. Raghavendra and Busch [24] proposed a sparse representation of the features scheme for the recognition of palmprint obtained from the Bank of Binary Features of the Statistics Images or B-BSIF. Meanwhile, Zhai et al. [13] proposed that the fusion between the block-wise bi-directional two-dimensional principal components analysis and the classification of grouping spares classification is effective. Ergen [56] introduced a method that integrates discrete cosine transformation (DCT) and an autoregressive (AR) modeling for biometric identification. In a study by Kumar and Wang[67], a convolutional neural network (CNN) was presented as a matcher left palmprint with right 
palmprint images of the same hand. They improved the similarity between the left palm and right palm of the same person. Fei et al. [68] performed a Gabor half-filter bank for the extraction of the double half of the palm recognition. In addition, they evaluated this work by comparing this method with a single dominant orientation. It was found that the double half-orientations could characterize the global orientation feature of a palmprint with greater precision. Zhang et al. [69] conducted a hybrid approach by combining the weighted adaptive center-symmetric local binary pattern (WACSLBP) with the weighted sparse representation (WSRC) to improve the classification. This approach consisted of coarse and fine steps. In the coarse step, the similarity between the test sample and one sample of each training class are being used. A small number of candidate classes of the test sample is reserved, and most of the training classes could be excluded. Whereas, in the fine step, the rotation invariant weighted histogram feature vector was extracted from each candidate's sample. Moreover, using WACS-LBP for the testing sample, the weighted sparse representation optimal problem was constructed using the similarity between the test sample and each candidate training sample. Table 5 summarizes previous works founded on the contact based database images.

\section{B. Review based Contactless Palmprint Database}

IITD and CASIA databases are examples of contactless images, which are collected by using a commercial camera under free environment using a non-user peg. Fig. 9 illustrates a contactless image. So the problems in this database are variants to translation, scaling, rotation, illumination, and noise. The contactless images are under low-resolution. Therefore, the line and texture are the most important features. A number of researchers use subspace learning approaches [68] and collaborative approach [71]. Robust features such as SIFT [72], LBP [73] LLDP [74], and OLOF [75] show excellent robustness to rotation and scaling, illumination and translation changes. These descriptors have achieved a good accuracy in contactless images. Fig. 10 and Fig. 11 illustrate SIFT and LBP features respectively. However, many algorithms for contactless recognition have been developed over the years. Kumar and Zhang [76] introduced the user's identity characterization through the simultaneous use of three main representations (hybrid approach) of the palmprint, namely Gabor features, line features, and principal component. Chu et al. [52] proposed a famous Gabor magnitude features. Kumar and Kumar [77] investigated the possibility of using the palmprint as a cryptic vault to develop a reliable and easy-touse encryption scheme that suggests the use of symmetric and asymmetric encryption approaches. Palanikumar et al. [78] introduced the contrast enhancement scheme called adaptive Increasing Value Histograms equalization (AIVHE) which can be used to adaptively match the Histograms equalization (HE) based on the palmprint lines, textures and hand geometry properties. Zhao et al.[79] used SIFT features and I-RANSAC algorithm for palmprint verification for the contactless dataset.
Kanchana and Balakrishnan [80] proposed a method of RabinKarp Palm-Print Pattern Matching (RPPM) to improve the matching accuracy of the palmprint features by using the double hash in RPPM. Ali et al. [25] used a hybrid approach for palmprint identification, by using various algorithms such as local binary pattern (LBP), two-dimensional localized preserving projection (2DLPP) and the fusion of LBP + 2DLPP. Table 6 summarizes previous works based on the contactless database.

a.

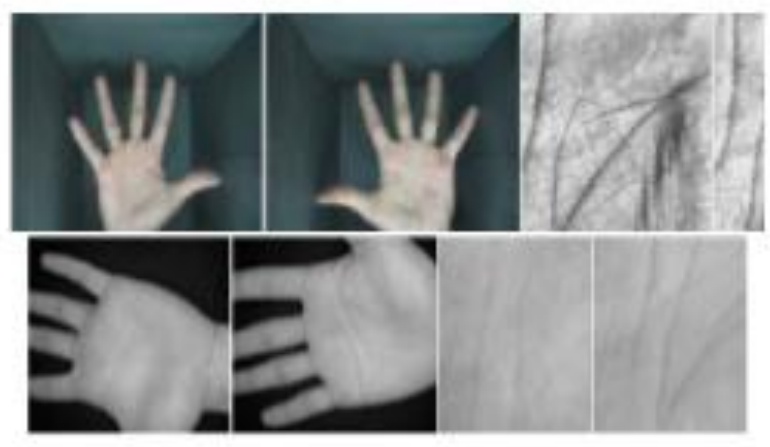

Fig. 9. Contactless Image a. IITD Images b. CASIA Images.

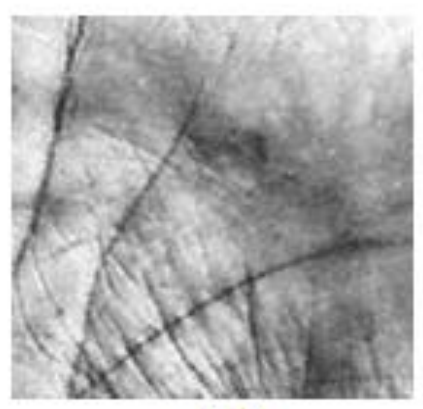

(a)

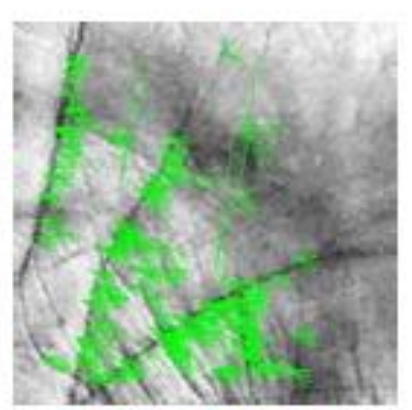

(b)
Fig. 10. Extract SIFT Features a. ROI b. SIFT Features.

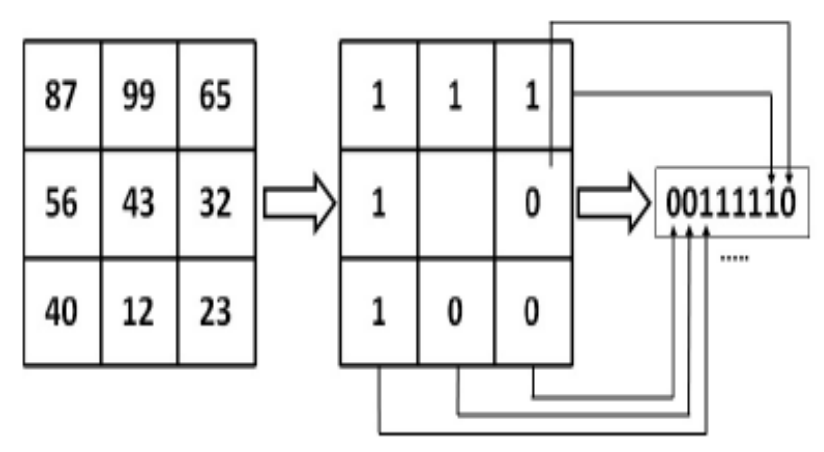

Fig. 11. Examples of LBP. 
TABLE VII. PREVIous Works BASED CONTACTLESS Database

\begin{tabular}{|c|c|c|c|}
\hline Reference & Recognition Approach & Parameter & Database \\
\hline \multirow{3}{*}{ [76] } & Gabor features & $\begin{array}{l}\mathrm{FAR}=0.82 \text { FRR }=7.20 \\
\mathrm{ERR}=4.89\end{array}$ & \multirow{3}{*}{$\begin{array}{l}\text { Unknown database of } 100 \text { users/. } 1000 \text { image, } \\
\text { acquired by a digital camera using peg-free setup }\end{array}$} \\
\hline & line Features & $\begin{array}{l}F A R=2.95 \\
F R R=7.60 \\
E R R=6.19\end{array}$ & \\
\hline & PCA features & $\begin{array}{c}\mathrm{FAR}=2.74 \\
\mathrm{FRR}=7.20 \\
\mathrm{ERR}=5.83\end{array}$ & \\
\hline \multirow{2}{*}[52]{} & \multirow{2}{*}{ Gabor + LDA } & $\mathrm{ERR}=0.35$ & UST database / 5560images \\
\hline & & $\mathrm{ERR}=0.17$ & CASIA / 4796 images \\
\hline [77] & DCT & $\mathrm{FAR}=0.35, \mathrm{ERR}=0.3$ & $\begin{array}{l}\text { Unknown database captured by a digital camera } \\
\text { without pinout in indoor environments / 85images }\end{array}$ \\
\hline [78]. & AIVHE & & $\begin{array}{l}\text { Unknown database captured by Nokia } 2700 \text { with } \\
1200 \times 1700 \text { resolution } / 100 \text { images }\end{array}$ \\
\hline [79]. & SIFT + I-RANSAC. & $\begin{array}{l}E R R=0.5134 \text { left palm } \\
E R R=0.5524 \text { Right palm }\end{array}$ & IIT Delhi's version 1.0 \\
\hline [80] & RPPM & $\mathrm{ACC}=73.88 \%$ & CASIA/35 user \\
\hline [53] & half-Gabor" filters & $\mathrm{EER}=0.0633$ & IITD database \\
\hline \multirow{3}{*}{ [25] } & LBP & $\begin{array}{l}\mathrm{ACC}=95.7 \% \quad \mathrm{FAR}=0.043 \\
\mathrm{FRR}=0.043 \\
\mathrm{ERR}=0.043\end{array}$ & \multirow{3}{*}{ CASIA / 1200 image } \\
\hline & 2DLPP & $\begin{array}{c}\mathrm{ACC}=97.33 \% \quad \mathrm{FAR}=0.030 \\
\mathrm{FRR}=0.023 \\
\mathrm{ERR}=0.0265\end{array}$ & \\
\hline & $\mathrm{LBP}+2 \mathrm{DLPP}$ & $\begin{array}{c}\mathrm{ACC}=98.55 \% \quad \mathrm{FAR}=0.0221 \\
\mathrm{FRR}=0.0145 \\
\mathrm{ERR}=0.0183\end{array}$ & \\
\hline [69] & WACSLBP and WSRC & $\mathrm{ACC}=99.14 \%$ & CASIA \\
\hline
\end{tabular}

\section{Review based High-Resolution Palmprint Images}

The high-resolution images are usually greater than 500dp [81]. These images include ridge and creaser features whereby the ridges are divided into local ridge direction (LRD) and minutia, while the creaser includes principle lines. Fig. 12 shows the features of high-resolution images from THUPALMLAB.

LRD is the angle between the ridge across the small area and the horizontal axis. (LRD) represents ( $(\mathrm{ij}$, rij), where $\Theta \mathrm{ij}$ is the ridge direction at $(\mathrm{i}, \mathrm{j}$ ) pixel and the (rij) represents the crosses ponding direction. [82].
To extract the LRD features, three methods have are widely used; gradient based, discrete Fourier transform and Gabor filter. In these methods, (rij) is calculated by deriving the direction vector in the local window. In a gradient based method, the orthogonal direction which represents the maximum changes in the intensity is calculated as the LRD [83].

In the DFT using the sine wave to represent the ridges in the palmprint image, DFT is considered as the most effective tool to extract the LRD feature [81]. Though, in the Gabor filter based, the LRD area is calculated by choosing the direction of the Gabor filter which performs the max-filtering response after applying multiple frequency spectrum in the 
local area [83]. However, the Gabor filter is considered as a complicated method that requires more time to execute.

Minutiae points are considered the most significant feature in high-resolution images [82]. Minutiae points are represented by a vector $(\mathrm{x}, \mathrm{y}, \Theta)$ where $\mathrm{x}, \mathrm{y}$ are the coordinates of a point and $\Theta$ is the LRD direction. To extract the minutia features, the following three steps are involved [23]:

1) Finding $\Theta$ of LRD by using Gabor filter;

2) Obtaining the Skelton ridge image by binarized and thinned palmprint image;

3) Calculating the center of the ridge of each pixel to detect minutiae points.

Principle lines feature can be seen in both low resolution and high-resolution images [84]. The principles line involves three long creases. The MFART and Hough transform is applied to detect these lines [84]. Fig. 13 shows these three features.

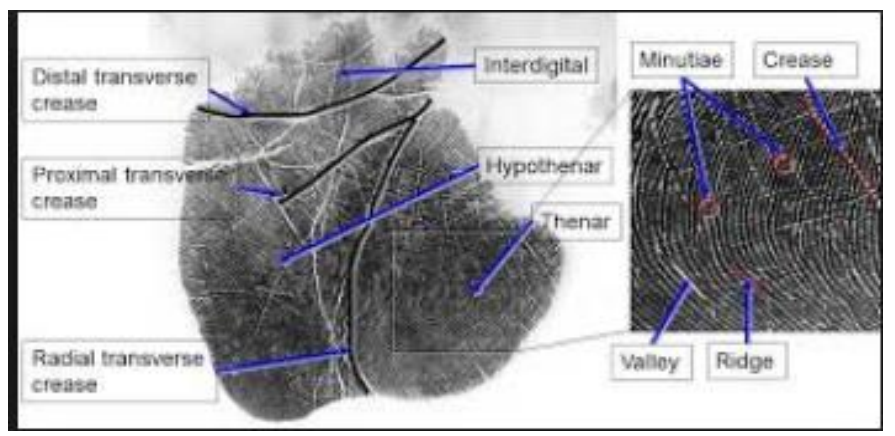

Fig. 12. High-Resolution Feature from THUPALMLAB Database.

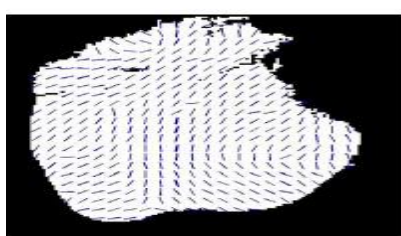

(a)

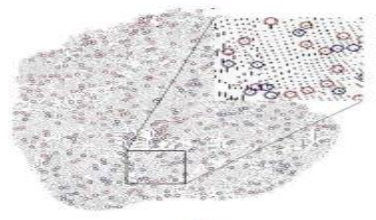

(c)

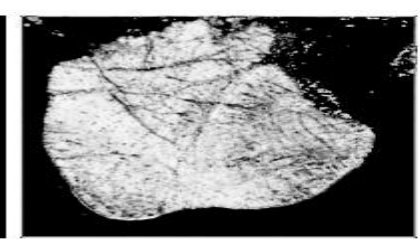

(b)

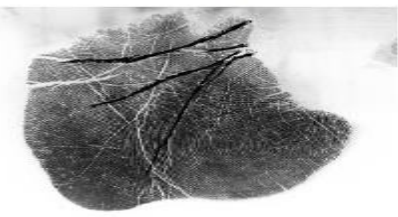

(d)
Fig. 13. Line Features: a. LRD Map b. Local Ridge Density Image, c. Minutiae Point Map, D. Principal Line Image.

On the other hand, Carreira et al. [85] used SIFT algorithm with the RGB-mapping algorithm. Dai and Zhou [84] used a multi feature-based matching technique that took 5 s to achieve a single match. Jain and Feng [81] used a minutiae code while Wang et al. [86] and Wang et al. [87] used the minutiae based method which performed local matching before global similarity. Cappelli et al. [88] extended the fingerprint encoding for palmprint images. These studies had achieved excellent results but have suffered from high computational complexity for a large number of minutiae points to be compared during matching. To solve this problem, Tariq et al.[22] conducted GPU to decrease the time of execution. The GPU is a special processor used in the graphic to perform true general-purpose. In addition, the processor requires recombining various software. They have a rudimentary programming tool as well as are poor in programming languages. Table 7 summarizes the previous works based on the high-resolution database.

TABLE VIII. PREVIOUS WORKS BASED High-RESOlUtion DATABASE

\begin{tabular}{|l|l|l|l|}
\hline Reference & $\begin{array}{l}\text { Recognition } \\
\text { Approach }\end{array}$ & Parameter & Database \\
\hline$[85]$ & SIFT & EER=22.5\% & THUPALMLAB \\
\hline$[22]$ & $\begin{array}{l}\text { Minutiae } \\
\text { encoding }\end{array}$ & EER=0.38 & THUPALMLAB \\
\hline$[20]$ & $\begin{array}{l}\text { RGB-mapping } \\
\text { algorithm }\end{array}$ & --- & THUPALMLAB \\
\hline$[84]$ & $\begin{array}{l}\text { Composite } \\
\text { algorithm }\end{array}$ & FRR=16\% & THUPALMLAB \\
\hline
\end{tabular}

$(\mathrm{ACC}=$ accuracy, $\mathrm{FAR}=$ false acceptance rate, $\mathrm{ERR}=$ error recognition rate, $\mathrm{FRR}=$ false rejection rate, $\mathrm{EER}=$ equal error rate)

\section{CONCLUSION}

This paper provides a comprehensive review of palmprint based biometric. We begin with investigating the biometric system. Then, we introduce an overview of palmprint technology; we summarize tables of the previous works based unimodal palmprint based on different databases. To sum up, palmprint recognition is still an open problem and not completely solved. For the importance of contactless images, accuracy needs improvement. Furthermore, the high-resolution images need to be investigated more as these images serve as an important source for high-security application for forensic usages. However, the unimodal biometric system has a variety of problems, such as; the noise, variance within the class; discrimination; nonuniversality and spoofing. To overcome the limitations of the unimodal system, a multimodal palmprint can be used by combining right and left palmprints at different levels of fusion. In the forthcoming paper, we will introduce an overview of multimodal palmprint biometric system.

\section{ACKNOWLEDGMENT}

The authors would like to thank the Ministry of Higher Education (MOHE) and University Technology Malaysia (UTM) for their educational and financial support. This work is conducted at the Razak Faculty of Technology and Informatics under Cyber-Physical Systems Research Group (CPS RG) and funded by Universiti Teknologi Malaysia (GUP Tier 1: Q. K130000.2538.18H2).

\section{REFERENCES}

[1] Gomez-Barrero, M., et al., General Framework to Evaluate Unlinkability in Biometric Template Protection Systems. IEEE Transactions on Information Forensics and Security, 2018. 13(6): p. 1406-1420.

[2] Naderi, H., B.H. Soleimani, and S. Matwin. Manifold Learning of Overcomplete Feature Spaces in a Multimodal Biometric Recognition 
System of Iris and Palmprint. in 2017 14th Conference on Computer and Robot Vision (CRV). 2017. IEEE.

[3] Deligne, S., et al., Methods and apparatus for use in speech recognition systems for identifying unknown words and for adding previously unknown words to vocabularies and grammars of speech recognition systems. 2017, Google Patents.

[4] Singh, D., et al., Real-time observation of DNA recognition and rejection by the RNA-guided endonuclease Cas9. Nature communications, 2016. 7: p. 12778.

[5] Fouad, K.M., B.M. Hassan, and M.F. Hassan, User authentication based on dynamic keystroke recognition. International Journal of Ambient Computing and Intelligence (IJACI), 2016. 7(2): p. 1-32.

[6] Biswas, S. and J. Sil, An efficient face recognition method using contourlet and curvelet transform. Journal of King Saud UniversityComputer and Information Sciences, 2017.

[7] Pillai, J.K., M. Puertas, and R. Chellappa, Cross-sensor iris recognition through kernel learning. IEEE transactions on pattern analysis and machine intelligence, 2014. 36(1): p. 73-85.

[8] Chan, A., T. Halevi, and N. Memon. Leap motion controller for authentication via hand geometry and gestures. in International Conference on Human Aspects of Information Security, Privacy, and Trust. 2015. Springer.

[9] Yuan, C., X. Sun, and R. Lv, Fingerprint liveness detection based on multi-scale LPQ and PCA. China Communications, 2016. 13(7): p. 6065.

[10] Makihara, Y., et al., Gait recognition: Databases, representations, and applications. Wiley Encyclopedia of Electrical and Electronics Engineering, 2015.

[11] Vera-Rodriguez, R., et al., DYNAMIC SIGNATURE RECOGNITION FOR AUTOMATIC STUDENT AUTHENTICATION. Departamento de Tecnologia Electronica y de las Comunicaciones, EPS, Universidad Autonoma de Madrid (SPAIN), 2015.

[12] Xin, Z.D.P., et al., Palmprint recognition based on deep learning. ICWMMN2015 Proceedings, 2015.

[13] Zhai, L., et al., An efficient classification method based on principal component and sparse representation. SpringerPlus, 2016. 5(1): p. 832.

[14] Jain, Y. and M. Juneja, A Novel Approach for Multimodal Biometric System Using Iris and PalmPrint, in Progress in Advanced Computing and Intelligent Engineering. 2018, Springer. p. 79-88, springer.

[15] Genovese, A., V. Piuri, and F. Scotti, Touchless palmprint recognition systems. Vol. 60. 2014: Springer.

[16] Gudavalli, M., S.V. Raju, and B. Chandrasekhar. An Optimal Content Matcher for Accurate Human Recognition in Multimodal Biometric Systems. in Advanced Computing (IACC), 2016 IEEE 6th International Conference on. 2016. IEEE.

[17] Srivastava, S., Accurate Human Recognition by Score-Level and FeatureLevel Fusion Using Palm-Phalanges Print. Arabian Journal for Science and Engineering, 2018. 43(2): p. 543-554.

[18] Barra, S., Design of a Multi-biometric Platform, based on physical traits and physiological measures: Face, Iris, Ear, ECG and EEG. 2016, Universita'degli Studi di Cagliari.

[19] Zhang, D., W. Zuo, and F. Yue, A comparative study of palmprint recognition algorithms. ACM computing surveys (CSUR), 2012. 44(1): p. 2.

[20] Rajeev, S., et al. Forensic print extraction using 3D technology and its processing. in Mobile Multimedia/Image Processing, Security, and Applications 2017. 2017. International Society for Optics and Photonics.

[21] Iula, A. and D. Nardiello, A Method for Biometric Recognition of Ultrasound Palmprint Based on Principal Lines. International Journal of Future Computer and Communication, 2016. 5(1): p. 13-17.

[22] Tariq, S.A., et al., Massively parallel palmprint identification system using GPU. Cluster Computing, 2017: p. 1-16,Springer Science+Business Media, LLC 2017.

[23] Fei, L., et al., Feature Extraction Methods for Palmprint Recognition: A Survey and Evaluation. IEEE Transactions on Systems, Man, and Cybernetics: Systems, 2018.
[24] Raghavendra, R. and C. Busch, Texture based features for robust palmprint recognition: a comparative study. EURASIP Journal on Information Security, 2015. 2015(1): p. 5.

[25] Ali, M.M.H., P.L. Yannawar, and A.T. Gaikwad, Multi-Algorithm of Palmprint Recognition System Based on Fusion of Local Binary Pattern and Two-Dimensional Locality Preserving Projection. Procedia Computer Science, 2017. 115(Supplement C): p. 482-492.

[26] ping Tian, D., A review on image feature extraction and representation techniques. International Journal of Multimedia and Ubiquitous Engineering, 2013. 8(4): p. 385-396.

[27] Hassaballah, M., A.A. Abdelmgeid, and H.A. Alshazly, Image Features Detection, Description and Matching, in Image Feature Detectors and Descriptors. 2016. p. 11-45.

[28] Loncomilla, P., J. Ruiz-del-Solar, and L. Martínez, Object recognition using local invariant features for robotic applications: A survey. Pattern Recognition, 2016. 60: p. 499-514.

[29] Li, Y., K. Wang, and D. Zhang. Palmprint recognition based on translation invariant Zernike moments and modular neural network. in International Symposium on Neural Networks. 2005. Springer.

[30] Noh, J.S. and K.H. Rhee. Palmprint identification algorithm using Hu invariant moments and Otsu binarization. in Computer and Information Science, 2005. Fourth Annual ACIS International Conference on. 2005. IEEE.

[31] Jia, W., et al., Histogram of oriented lines for palmprint recognition. IEEE Transactions on Systems, Man, and Cybernetics: Systems, 2014. 44(3): p. 385-395.

[32] Morales, A., M.A. Ferrer, and A. Kumar. Improved palmprint authentication using contactless imaging. in Biometrics: Theory Applications and Systems (BTAS), 2010 Fourth IEEE International Conference on. 2010. IEEE.

[33] Badrinath, G. and P. Gupta. Robust biometric system using palmprint for personal verification. in International Conference on Biometrics. 2009. Springer.

[34] Guo, Z., et al. Hierarchical multiscale LBP for face and palmprint recognition. in Image Processing (ICIP), 2010 17th IEEE International Conference on. 2010. IEEE.

[35] ZHANGH, L. and Y. LIU, Improved binary robust invariant scalable keypoints algorithm fusing depth information. Journal of Computer Applications, 2015. 35(8): p. 2285-2290.

[36] Kang, W. and X. Chen, Fast representation based on a double orientation histogram for local image descriptors. IEEE Transactions on Image Processing, 2015. 24(10): p. 2915-2927.

[37] Kong, A., D. Zhang, and M. Kamel, A survey of palmprint recognition. Pattern Recognit, 2009. 42.

[38] Lumini, A. and L. Nanni, Overview of the combination of biometric matchers. Information Fusion, 2017. 33: p. 71-85.

[39] Wu, X., D. Zhang, and K. Wang, Fisherpalms based palmprint recognition. Pattern recognition letters, 2003. 24(15): p. 2829-2838.

[40] Sun, X., M. Chen, and A. Hauptmann. Action recognition via local descriptors and holistic features. in Computer Vision and Pattern Recognition Workshops, 2009. CVPR Workshops 2009. IEEE Computer Society Conference on. 2009. IEEE.

[41] Shang, L., et al., Palmprint recognition using FastICA algorithm and radial basis probabilistic neural network. Neurocomputing, 2006. 69(1315): p. 1782-1786.

[42] Jaswal, G., R. Nath, and A. Kaul. Texture based palm Print recognition using 2-D Gabor filter and sub space approaches. in Signal Processing, Computing and Control (ISPCC), 2015 International Conference on. 2015. IEEE.

[43] Ekinci, M. and M. Aykut, Kernel Fisher discriminant analysis of Gabor features for online palmprint verification. Turkish Journal of Electrical Engineering \& Computer Sciences, 2016. 24(2).

[44] Kusban, M., A. Susanto, and O. Wahyunggoro, Combination a Skeleton Filter and Reduction Dimension of Kernel PCA Based on Palmprint Recognition. International Journal of Electrical and Computer Engineering, 2016. 6(6): p. 3255.

[45] Alsubari, A. and R. Ramteke, Extraction of Face and Palmprint Features based on LBP, HOG and Zernike Moments. Extraction, 2017. 172(5). 
[46] Liu, Y., Y. Yin, and S. Zhang. Hand Gesture Recognition Based on HU Moments in Interaction of Virtual Reality. in 2012 4th International Conference on Intelligent Human-Machine Systems and Cybernetics. 2012.

[47] Saranraj, S., et al. Palm print biometric recognition based on Scattering Wavelet Transform. in 2016 International Conference on Wireless Communications, Signal Processing and Networking (WiSPNET). 2016.

[48] Hennings, P., M. Savvides, and B.V.K.V. Kumar. Palmprint Recognition with Multiple Correlation Filters Using Edge Detection for Class-Specific Segmentation. in 2007 IEEE Workshop on Automatic Identification Advanced Technologies. 2007.

[49] Mu, M., Q. Ruan, and Y. Ming. Shape parameters of Gaussian as descriptor for palmprint recognition based on Dual-tree Complex Wavelet Transform. in IEEE 10th INTERNATIONAL CONFERENCE ON SIGNAL PROCESSING PROCEEDINGS. 2010.

[50] Pudzs, M., et al. Complex matched filter for line detection. in 2015 9th International Symposium on Image and Signal Processing and Analysis (ISPA). 2015.

[51] Fang, L. and M.K.H. Leung. Palmprint matching using pairwise relative angle based Hausdorff distance. in 2009 IEEE 13th International Symposium on Consumer Electronics. 2009.

[52] Chu, R., et al. Learning Gabor magnitude features for palmprint recognition. in Asian Conference on Computer Vision. 2007. Springer.

[53] Fei, L., Y. Xu, and D. Zhang, Half-orientation extraction of palmprint features. Pattern Recognition Letters, 2016. 69: p. 35-41.

[54] Jia, W., D.-S. Huang, and D. Zhang, Palmprint verification based on robust line orientation code. Pattern Recognition, 2008. 41(5): p. 15041513.

[55] ALMEZOGHY, H.H., et al., HUMAN IDENTIFICATION WITH PALM PRINT BASED ON LOCAL BINARY PATTERN. 2017.

[56] Ergen, B., Scale invariant and fixed-length feature extraction by integrating discrete cosine transform and autoregressive signal modeling for palmprint identification. Turkish Journal of Electrical Engineering \& Computer Sciences, 2016. 24: p. 1768-1781.

[57] Doush, I.A. and A.-B. Sahar, Currency recognition using a smartphone: comparison between color SIFT and gray scale SIFT algorithms. Journal of King Saud University-Computer and Information Sciences, 2017. 29(4): p. 484-492.

[58] Zhang, L., H. Li, and J. Niu, Fragile bits in palmprint recognition. IEEE Signal processing letters, 2012. 19(10): p. 663-666.

[59] Bounneche, M.D., et al., Multi-spectral palmprint recognition based on oriented multiscale log-Gabor filters. Neurocomputing, 2016. 205: p. 274-286.

[60] Malik, J., et al., Accuracy improvement in palmprint authentication system. International Journal of Image, Graphics and Signal Processing, 2015. 7(4): p. 51.

[61] Zhang, D., et al., Online palmprint identification. IEEE Transactions on pattern analysis and machine intelligence, 2003. 25(9): p. 1041-1050.

[62] Zuo, W., et al. The multiscale competitive code via sparse representation for palmprint verification. in Computer Vision and Pattern Recognition (CVPR), 2010 IEEE Conference on. 2010. IEEE.

[63] Guo, Z., et al., Palmprint verification using binary orientation cooccurrence vector. Pattern Recognition Letters, 2009. 30(13): p. 12191227.

[64] Fei, L., et al., Double-orientation code and nonlinear matching scheme for palmprint recognition. Pattern Recognition, 2016. 49: p. 89-101.

[65] Raut, S.D. and V.T. Humbe, Biometric Palm Prints Feature Matching for Person Identification. International Journal of Modern Education and Computer Science, 2012. 4(11): p. 61-69.

[66] Li, H., L. Wang, and Z. Zhang, Robust Palmprint Recognition Based on Directional Representations. Intelligent Information Processing VI, 2012: p. $372-381$.

[67] Kumar, A. and K. Wang, Identifying humans by matching their left palmprint with right palmprint images using convolutional neural network. Proc. DLPR, 2016.
[68] Fei, L., et al., Low-rank representation integrated with principal line distance for contactless palmprint recognition. Neurocomputing, 2016. 218: p. 264-275.

[69] Zhang, S., et al., Combining Modified LBP and Weighted SRC for Palmprint Recognition. Signal, Image and Video Processing, 2018: p. 18.

[70] Qu, Z. and Z.Y. Wang, The Improved Algorithm of Scale Invariant Feature Transform on Palmprint Recognition. Advanced Materials Research, 2011. 186: p. 565-569.

[71] Zhang, L., et al., Towards contactless palmprint recognition: A novel device, a new benchmark, and a collaborative representation based identification approach. Pattern Recognition, 2017. 69: p. 199-212.

[72] Morales, A., M.A. Ferrer, and A. Kumar, Towards contactless palmprint authentication. IET computer vision, 2011. 5(6): p. 407-416.

[73] Ojala, T., M. Pietikainen, and T. Maenpaa, Multiresolution gray-scale and rotation invariant texture classification with local binary patterns. IEEE Transactions on pattern analysis and machine intelligence, 2002. 24(7): p. 971-987.

[74] Luo, Y.-T., et al., Local line directional pattern for palmprint recognition. Pattern Recognition, 2016. 50: p. 26-44.

[75] Sun, Z., et al. Ordinal palmprint represention for personal identification [represention read representation]. in Computer Vision and Pattern Recognition, 2005. CVPR 2005. IEEE Computer Society Conference on. 2005. IEEE.

[76] Kumar, A. and D. Zhang, Personal authentication using multiple palmprint representation. Pattern Recognition, 2005. 38(10): p. 16951704.

[77] Kumar, A. and A. Kumar, Development of a New Cryptographic Construct Using Palmprint-Based Fuzzy Vault. EURASIP Journal on Advances in Signal Processing, 2009. 2009(1): p. 967046.

[78] Palanikumar, S., M. Sasikumar, and J. Rajeesh, Entropy Optimized Palmprint Enhancement Using Genetic Algorithm and Histogram Equalization. International Journal of Genetic Engineering, 2012. 2(2): p. 12-18.

[79] Zhao, Q., X. Wu, and W. Bu. Contactless palmprint verification based on SIFT and iterative RANSAC. in Image Processing (ICIP), 2013 20th IEEE International Conference on. 2013. IEEE.

[80] Kanchana, S. and G. Balakrishnan, Palm-Print Pattern Matching Based on Features Using Rabin-Karp for Person Identification. The Scientific World Journal, 2015. 2015: p. 8, Hindawi Publishing Corporation.

[81] Jain, A.K. and J. Feng, Latent palmprint matching. IEEE Transactions on Pattern Analysis and Machine Intelligence, 2009. 31(6): p. 1032-1047.

[82] Maltoni, D., et al., Handbook of fingerprint recognition. 2009: Springer Science \& Business Media.

[83] Jain, A.K., S. Prabhakar, and L. Hong, A multichannel approach to fingerprint classification. IEEE transactions on pattern analysis and machine intelligence, 1999. 21(4): p. 348-359.

[84] Dai, J. and J. Zhou, Multifeature-based high-resolution palmprint recognition. IEEE Transactions on Pattern Analysis and Machine Intelligence, 2011. 33(5): p. 945-957.

[85] Carreira, L., et al., Personal identification from degraded and incomplete high resolution palmprints. IET Biometrics, 2015. 4(2): p. 53-61.

[86] Wang, R., et al. Towards regional fusion for high-resolution palmprint recognition. in Graphics, Patterns and Images (SIBGRAPI), 2013 26th SIBGRAPI-Conference on. 2013. IEEE.

[87] Wang, R., et al., Regional fusion for high-resolution palmprint recognition using spectral minutiae representation. IET biometrics, 2014. 3(2): p. 94-100.

[88] Cappelli, R., M. Ferrara, and D. Maio, A fast and accurate palmprint recognition system based on minutiae. IEEE Transactions on Systems, Man, and Cybernetics, Part B (Cybernetics), 2012. 42(3): p. 956-962. 